\title{
Fact Sheet
}

\section{Millions of Adults Ages 50 and Older Rely on the Supplemental Nutrition Assistance Program (SNAP)}

Olivia Dean and Carlos Figueiredo

AARP Public Policy Institute

The Supplemental Nutrition Assistance Program (SNAP) is the nation's largest antihunger program and a lifeline for millions of people, including adults ages 50 and older, who are at risk for food insecurity (i.e., limited or uncertain access to adequate food). ${ }^{1}$ SNAP provides financial assistance to low-income individuals and families to help buy the food they need. The program reduces food insecurity and poverty and is linked to improved health outcomes, including reduced hospitalizations among low-income older adults. ${ }^{2}$

Because SNAP is designed to respond to the needs of lowincome populations, it has been a particularly important program during the COVID-19 pandemic. Millions of people enrolled in SNAP during the pandemic and associated economic downturn, ${ }^{3}$ which likely played a role in keeping the share of adults ages 50 and older who were food-insecure unchanged at 8 percent (or 9.5 million people) during that time period. ${ }^{4}$

This Fact Sheet describes selected characteristics of adults ages 50 and older who participate in SNAP and the benefits their households receive. ${ }^{5}$ The paper includes analysis of SNAP participants ages 50 to 59 as well as those ages 60 and older, and state-level data are included in the appendices.

\section{Characteristics of Older Adults Participating in SNAP}

\section{Almost Half of SNAP Households Have an Older Adult}

In 2019, 46 percent (8.7 million) of all SNAP households included at least one adult age 50 or older. That share varied by state and territory, from 30 percent in Utah to 58 percent in Florida and New York (appendix A, table 1). While the number of SNAP households with at least one adult age 50 or older stayed about the same between 2018 and 2019, the share increased slightly from 2018 (from 44 percent) due to a decrease in overall SNAP participation.

\section{What Is a SNAP \\ Household?}

Eligibility for SNAP is

determined at the household

level. A household can

be composed of a single

individual or a group of

people who live together and

purchase and prepare food

together. For the program's

determination of household

eligibility, the household

typically must apply using

aggregate income, expenses, and assets. ${ }^{6}$ 
The number of SNAP households with adults ages 50 to 59 decreased from about 3.8 million in 2018 to 3.6 million in 2019, but the overall share stayed the same. Conversely, the number of SNAP households with adults ages 60 and older increased from 5.1 million in 2018 to nearly 5.3 million in 2019. As a result, the share of households with adults 60 or older rose from 26 percent in 2018 to 28 percent in 2019.

\section{Most Older SNAP Enrollees Live Alone}

Older SNAP participants are more likely than younger SNAP participants to live alone. In 2019, 77 percent of SNAP households with an adult age 50 and older were single-person households, compared to 30 percent of younger households (without any person age 50 or older). Households with adults ages 50 and older had an average SNAP household size of 1.3 people, compared to 2.6 among households without any adult age 50 and older. ${ }^{7}$ Among older households, household size varied by state and territory, from 1.1 people in Connecticut to 1.8 in Alaska and 2.1 people in Guam (appendix A, table 2).
Households with adults ages 60 and older were most likely to be the smallest, with an average household size of 1.2 people compared to 1.5 people among households with adults ages 50 to 59.

\section{Most Older SNAP Households Live below the Poverty Line}

SNAP households must have incomes below a certain amount to be eligible for benefits. The average gross monthly income of SNAP households with at least one adult age 50 or older was about $\$ 860$ in 2019 , or $\$ 10,316$ a year (appendix A, table 2). Three-quarters (75 percent) of the 8.7 million SNAP households with adults ages 50 and older had incomes below the federal poverty level (FPL), which in 2019 was $\$ 12,490$ a year for a one-person household and $\$ 16,910$ a year for a two-person household. The Virgin Islands had the highest share of older SNAP households in poverty (92 percent; appendix A, table 2), while Vermont had the lowest (53 percent).

About one-sixth (16 percent) of SNAP participants ages 50 to 59 were reported to be working in 2019, compared to 5 percent of

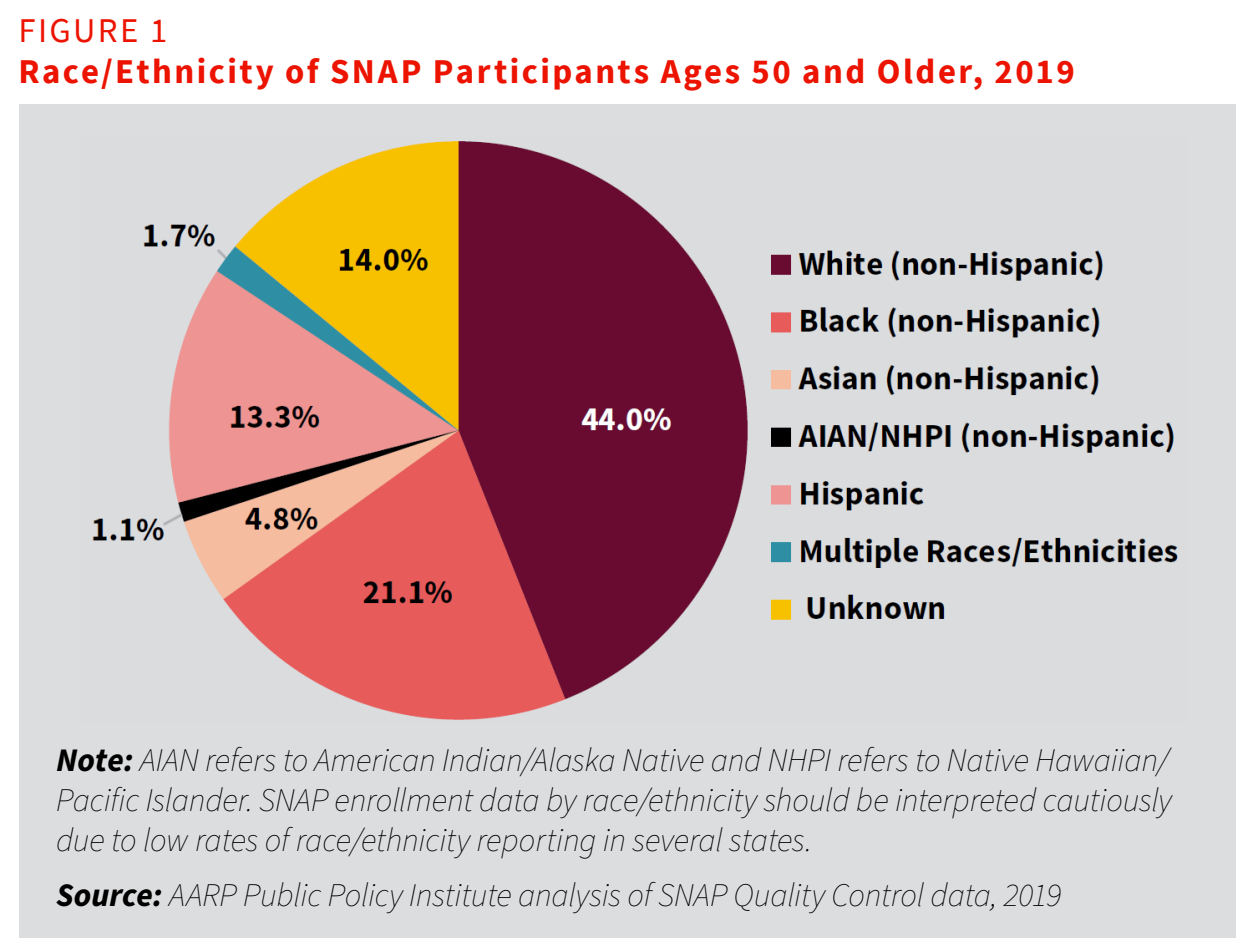


those ages 60 and older. However, recording employment at a given point in time overstates joblessness because many SNAP participants have unstable or temporary work and may be working on and off throughout the year. ${ }^{8}$ Relatively low employment rates among adults ages 50 to 59 could also be driven by high rates of disability (see section below).

\section{Nearly Half of SNAP Participants Ages 50 to 59 Have a Disability}

Food insecurity tends to be more common and more severe among SNAP households affected by disabilities, making these households some of the most vulnerable. ${ }^{9}$ Older SNAP participants are also more likely to have disabilities than younger participants. At the individual level, nearly half -47 percent-of the 3.7 million SNAP participants ages 50 to 59 had a disability in $2019 .{ }^{10}$ Among SNAP participants under age 50 , the share with disabilities was only about 9 percent. Half (49 percent) of SNAP households with adults ages 50 to 59 included at least one person age 50 to 59 with a disability.

\section{Older SNAP Recipients Represent Different Races and Ethnicities}

Among SNAP participants ages 50 and older, 13 percent are Hispanic, 44 percent are non-
Hispanic White, 21 percent are non-Hispanic Black, 5 percent are non-Hispanic Asian, 1 percent are non-Hispanic American Indian/ Alaska Native or Native Hawaiian/Pacific Islander, and 14 percent are of unknown race/ ethnicity (figure 1). Race/ethnicity data are unavailable for 14 percent of SNAP participants ages 50 and older due to low rates of race/ ethnicity reporting in several states.

\section{SNAP Benefits}

\section{SNAP Benefits Are Based on a Very Low-Cost Food Plan}

SNAP benefits are calculated using the US Department of Agriculture's (USDA's) Thrifty Food Plan (TFP), a very low-cost food plan that is supposed to be based on average consumption while meeting adequate nutrition and price targets. The TFP varies by household size, with larger SNAP households receiving larger benefit amounts. SNAP households are expected to spend about 30 percent of their net income on food. Thus, a monthly benefit allotment is calculated by multiplying net monthly income by 0.3 and subtracting that amount from the maximum monthly allotment for household size.

\section{SNAP Benefit Amounts Changed During Pandemic ${ }^{11}$}

Mary is 65 years old, lives alone, and receives $\$ 500$ in Social Security income per month. In October

2020 , she would have normally received \$54 in prepandemic monthly SNAP benefits. However, with pandemic emergency allotments, she received \$204. This amount increased to \$234 in January 2021 due to another temporary 15 percent boost. In October 2021, the 15 percent boost expired, but at the same time, a reevaluation of the USDA's Thrifty Food Plan-the basis on which SNAP benefits are calculatedtook effect. As a result, her SNAP benefit increased from \$234 to \$250 per month. When emergency allotments end, her monthly benefit will decrease to $\$ 100$, which is more than the $\$ 54$ she received before the pandemic because of the TFP reevaluation. 


\section{Nearly One-Third of Older SNAP Households Receive the Maximum Benefit}

Under normal SNAP rules, SNAP households with no net income receive the maximum SNAP benefit allotment for their household size (see maximum allotments by household size in appendix B). ${ }^{12}$ These lowest-income households do not have disposable income available to purchase food and often rely solely on SNAP.

In 2019, nearly a third (29 percent) of households with adult(s) ages 50 or older received the maximum benefit (appendix A, table 3). This share varied by state, from 15 percent in Hawaii to 51 percent in New York. Among households without an older adult, a larger share received the maximum benefit (43 percent).

On average, SNAP households with at least one adult age 50 and older received $\$ 142$ per month in 2019, or $\$ 1.56$ per meal (appendix A, table 3). ${ }^{13}$ The maximum benefit of $\$ 192$ in 2019 for a one-person household equates to approximately $\$ 2.10$ per meal (appendix B). ${ }^{14}$ However, in 2019, people who were food insecure reported spending an average $\$ 3.13$ per meal. ${ }^{15}$ Thus, even the maximum SNAP benefit was likely insufficient to cover all meal costs even before the pandemic caused an economic downturn.

\section{One in Six Older SNAP Households Receive the Minimum Benefit}

The federal government has established a nominal SNAP benefit floor. In 2019, that minimum SNAP benefit was $\$ 16$ per month for one- and two-person households in most states and the District of Columbia. ${ }^{16}$ Beginning in October 2021, the minimum monthly benefit increased to $\$ 20$ for most states. ${ }^{17}$

While most SNAP households with adults ages 50 and older receive more than the minimum benefit, 17 percent received the minimum benefit in 2019. The share receiving the minimum benefit varied by state and territory, from 1 percent in Guam and Hawaii to 34 percent in Wisconsin (appendix A, table 3). Older SNAP households were more likely than younger SNAP households to receive the minimum benefit. Among households without adults ages 50 and older, only 4 percent received the minimum benefit. In fact, of all SNAP households receiving the minimum benefit, three-quarters (77 percent) were households with adults ages 50 and older.

Households that qualify for the minimum benefit are still low-income households that must meet SNAP income requirements. Under typical SNAP rules, gross income must not be higher than 130 percent of the FPL (about $\$ 16,700$ for a single-person household in 2021), while net income must not be higher than 100 percent of the FPL. However, under federal rules, households with people ages 60 and older or people with disabilities need to meet only the net income limit. While the minimum benefit can help, it is a small supplement that covers only about five meals a month. ${ }^{18}$

\section{Fewer Than One in Six SNAP Households with Adults 60 and Older Claim the Medical Expense Deduction}

SNAP allows households that include adults ages 60 and older or people with disabilities to deduct medical expenses from their income for SNAP benefit calculation purposes. Any medical expenses over $\$ 35$ a month that have not otherwise been reimbursed can be deducted, which can help these households qualify for higher SNAP benefits. There are a wide range of allowable deductions, including health insurance premiums and copayments, prescription drug costs, dental and hearing costs, and transportation to medical appointments. ${ }^{19}$ Some states allow additional expenses not listed in federal regulations, such as home modifications and alternative therapies.

Many seniors face significant out-of-pocket medical costs, ${ }^{20}$ but only 15 percent of SNAP households with at least one adult age 60 or older claim the medical expense deduction. This share varies widely by state and territory, from just 4 percent in Maine and Washington, DC, to 41 percent in North Dakota and 69 percent in the Virgin Islands (appendix 
A, table 4). One of the potential reasons why the share claiming the medical deduction is so low is that medical expenses must be verified. To reduce the administrative burden on caseworkers and SNAP participants, some states use a standard medical deduction (set amount) when participants can prove medical expenses over $\$ 35$.

\section{A Critical Program for Older Adults, Especially during the COVID-19 Pandemic}

SNAP is an essential federal nutrition program that helps millions of older adults put food on the table. A countercyclical program, SNAP is designed to expand when the economy weakens and contract when the economy strengthens. Thus, the program has taken on heightened importance during the COVID-19 pandemic, as factors such as increased unemployment have cut into people's incomes and higher food prices have made it even more difficult for many low-income older adults to afford food. ${ }^{21}$
Federal and state governments temporarily increased SNAP benefits during the COVID-19 pandemic in response to the economic downturn. Several legislative and policy changes have occurred since March 2020, and as of October 2021 SNAP households are still seeing a modest increase of about $\$ 12$ to $\$ 16$ per month. ${ }^{22}$ Average benefits will drop once pandemic emergency allotments end but will remain higher than prepandemic benefits due to the Thrifty Food Plan reevaluation.

Between fiscal years 2019 and 2020, over 4 million more people of all ages (in 2.6 million households) enrolled in SNAP. ${ }^{23}$ SNAP enrollment has continued to climb since, and as of October 2021 over 6 million more people were enrolled in SNAP than in fiscal year 2019. ${ }^{24}$ Data presented in this report demonstrate a significant prepandemic need for SNAP among households with older adults, and the need has only increased during the pandemic. 


\section{Appendix A: State-Level Tables}

TABLE 1

SNAP Enrollment among Households with Adult(s) Ages 50 and Older, 2019

\begin{tabular}{|c|c|c|c|c|c|}
\hline State & $\begin{array}{c}\text { Total SNAP } \\
\text { Households } \\
\text { (All Ages) }\end{array}$ & $\begin{array}{l}\text { Total Households } \\
\text { with Adult(s) } 50+\end{array}$ & $\begin{array}{c}\text { Households } \\
\text { with } \\
\text { Adult(s) 50-59 }\end{array}$ & $\begin{array}{l}\text { Households } \\
\text { with } \\
\text { Adult(s) } 60+\end{array}$ & $\begin{array}{l}\text { Share of All SNAP } \\
\text { Households with } \\
\text { Adult(s) } 50+\end{array}$ \\
\hline Alabama & 336,117 & 149,402 & 75,110 & 78,655 & $44 \%$ \\
\hline Alaska & 36,106 & 16,008 & 8,385 & 8,537 & $44 \%$ \\
\hline Arizona & 368,321 & 158,520 & 73,114 & 91,727 & $43 \%$ \\
\hline Arkansas & 154,330 & 58,542 & 29,120 & 29,934 & $38 \%$ \\
\hline California & $1,912,235$ & 695,495 & 313,915 & 395,667 & $36 \%$ \\
\hline Colorado & 222,621 & 103,965 & 40,426 & 65,638 & $47 \%$ \\
\hline Connecticut & 208,386 & 101,375 & 37,367 & 64,979 & $49 \%$ \\
\hline Delaware & 62,376 & 31,363 & 17,361 & 14,629 & $50 \%$ \\
\hline District of Columbia & 64,967 & 29,885 & 14,090 & 16,017 & $46 \%$ \\
\hline Florida & $1,522,372$ & 877,574 & 290,415 & 611,085 & $58 \%$ \\
\hline Georgia & 635,011 & 255,847 & 102,461 & 158,466 & $40 \%$ \\
\hline Hawaii & 79,688 & 40,612 & 14,798 & 26,826 & $51 \%$ \\
\hline Idaho & 66,179 & 26,731 & 12,143 & 14,968 & $40 \%$ \\
\hline Illinois & 867,365 & 371,292 & 157,461 & 222,594 & $43 \%$ \\
\hline Indiana & 249,918 & 102,358 & 46,191 & 57,575 & $41 \%$ \\
\hline lowa & 149,892 & 53,790 & 28,544 & 26,539 & $36 \%$ \\
\hline Kansas & 90,508 & 38,974 & 17,555 & 22,228 & $43 \%$ \\
\hline Kentucky & 238,005 & 107,696 & 54,519 & 56,439 & $45 \%$ \\
\hline Louisiana & 371,079 & 152,250 & 78,280 & 74,706 & $41 \%$ \\
\hline Maine & 81,831 & 43,670 & 17,177 & 27,253 & $53 \%$ \\
\hline Maryland & 331,408 & 172,173 & 63,400 & 112,483 & $52 \%$ \\
\hline Massachusetts & 445,234 & 231,241 & 82,981 & 150,347 & $52 \%$ \\
\hline Michigan & 610,601 & 306,474 & 149,090 & 164,390 & $50 \%$ \\
\hline Minnesota & 203,969 & 88,613 & 38,873 & 50,663 & $43 \%$ \\
\hline Mississippi & 210,106 & 90,353 & 47,428 & 44,728 & $43 \%$ \\
\hline Missouri & 316,872 & 138,335 & 64,548 & 76,607 & $44 \%$ \\
\hline Montana & 51,744 & 23,908 & 10,797 & 13,506 & $46 \%$ \\
\hline Nebraska & 71,454 & 28,777 & 12,313 & 17,070 & $40 \%$ \\
\hline Nevada & 217,684 & 92,633 & 36,677 & 56,471 & $43 \%$ \\
\hline New Hampshire & 39,044 & 18,098 & 9,212 & 9,480 & $46 \%$ \\
\hline New Jersey & 353,632 & 198,163 & 58,939 & 144,350 & $56 \%$ \\
\hline New Mexico & 213,873 & 78,108 & 40,738 & 39,514 & $37 \%$ \\
\hline New York & $1,483,787$ & 858,528 & 267,974 & 605,022 & $58 \%$ \\
\hline North Carolina & 614,748 & 266,512 & 112,453 & 162,720 & $43 \%$ \\
\hline North Dakota & 23,176 & 9,863 & 3,865 & 6,064 & $43 \%$ \\
\hline Ohio & 675,942 & 335,812 & 158,873 & 184,651 & $50 \%$ \\
\hline Oklahoma & 258,127 & 108,285 & 56,713 & 54,464 & $42 \%$ \\
\hline Oregon & 346,536 & 175,195 & 70,197 & 108,769 & $51 \%$ \\
\hline Pennsylvania & 934,629 & 475,763 & 186,458 & 292,582 & $51 \%$ \\
\hline Rhode Island & 85,936 & 43,846 & 14,728 & 29,533 & $51 \%$ \\
\hline South Carolina & 275,813 & 123,985 & 60,209 & 65,559 & $45 \%$ \\
\hline South Dakota & 37,657 & 15,489 & 6,935 & 8,748 & $41 \%$ \\
\hline Tennessee & 420,474 & 187,076 & 94,290 & 98,720 & $44 \%$ \\
\hline Texas & $1,440,379$ & 579,092 & 234,012 & 346,945 & $40 \%$ \\
\hline Utah & 71,797 & 21,368 & 10,094 & 11,650 & $30 \%$ \\
\hline Vermont & 38,978 & 22,247 & 8,262 & 14,316 & $57 \%$ \\
\hline Virginia & 328,254 & 158,160 & 73,290 & 86,648 & $48 \%$ \\
\hline Washington & 473,593 & 206,844 & 75,913 & 133,261 & $44 \%$ \\
\hline West Virginia & 158,503 & 82,573 & 40,319 & 45,191 & $52 \%$ \\
\hline Wisconsin & 312,535 & 141,935 & 63,500 & 80,860 & $45 \%$ \\
\hline Wyoming & 11,755 & 4,598 & 2,413 & 2,226 & $39 \%$ \\
\hline Guam & 15,293 & 6,203 & 2,728 & 3,727 & $41 \%$ \\
\hline Virgin Islands & 11,160 & 5,072 & 1,724 & 3,465 & $45 \%$ \\
\hline United States & $18,802,000$ & $8,710,673$ & $3,588,375$ & $5,289,192$ & $46 \%$ \\
\hline
\end{tabular}

Note: SNAP households with adults 50+, 50-59, and 60+ refer to households with at least one eligible adult 50+, 50-59, and 60+, respectively.

Source: AARP Public Policy Institute analysis of SNAP Quality Control data, 2019 
TABLE 2

Characteristics of SNAP Households with Adult(s) Ages 50 and Older, 2019

\begin{tabular}{|c|c|c|c|c|}
\hline State & $\begin{array}{c}\text { Average } \\
\text { Household Size }\end{array}$ & $\begin{array}{l}\text { Single-Person } \\
\text { Households }\end{array}$ & $\begin{array}{l}\text { Average Gross Monthly } \\
\text { Household Income }\end{array}$ & $\begin{array}{l}\text { Share of Households with Gross } \\
\text { Income under Poverty Line }\end{array}$ \\
\hline Alabama & 1.4 & $80 \%$ & $\$ 812$ & $84 \%$ \\
\hline Alaska & 1.8 & $66 \%$ & $\$ 1,039$ & $75 \%$ \\
\hline Arizona & 1.5 & $72 \%$ & $\$ 822$ & $77 \%$ \\
\hline Arkansas & 1.4 & $75 \%$ & $\$ 783$ & $83 \%$ \\
\hline California & 1.4 & $70 \%$ & $\$ 805$ & $71 \%$ \\
\hline Colorado & 1.2 & $84 \%$ & $\$ 855$ & $74 \%$ \\
\hline Connecticut & 1.1 & $89 \%$ & $\$ 898$ & $66 \%$ \\
\hline Delaware & 1.5 & $70 \%$ & $\$ 921$ & $75 \%$ \\
\hline District of Columbia & 1.2 & $84 \%$ & $\$ 682$ & $85 \%$ \\
\hline Florida & 1.3 & $78 \%$ & $\$ 852$ & $76 \%$ \\
\hline Georgia & 1.4 & $75 \%$ & $\$ 846$ & $75 \%$ \\
\hline Hawaii & 1.4 & $56 \%$ & $\$ 894$ & $85 \%$ \\
\hline Idaho & 1.4 & $77 \%$ & $\$ 919$ & $77 \%$ \\
\hline Illinois & 1.3 & $80 \%$ & $\$ 875$ & $71 \%$ \\
\hline Indiana & 1.3 & $84 \%$ & $\$ 798$ & $81 \%$ \\
\hline lowa & 1.2 & $86 \%$ & $\$ 881$ & $69 \%$ \\
\hline Kansas & 1.3 & $67 \%$ & $\$ 806$ & $82 \%$ \\
\hline Kentucky & 1.4 & $53 \%$ & $\$ 699$ & $89 \%$ \\
\hline Louisiana & 1.3 & $80 \%$ & $\$ 724$ & $89 \%$ \\
\hline Maine & 1.3 & $81 \%$ & $\$ 1,024$ & $64 \%$ \\
\hline Maryland & 1.2 & $84 \%$ & $\$ 871$ & $73 \%$ \\
\hline Massachusetts & 1.2 & $81 \%$ & $\$ 989$ & $69 \%$ \\
\hline Michigan & 1.2 & $84 \%$ & $\$ 899$ & $71 \%$ \\
\hline Minnesota & 1.3 & $85 \%$ & $\$ 980$ & $70 \%$ \\
\hline Mississippi & 1.3 & $81 \%$ & $\$ 755$ & $86 \%$ \\
\hline Missouri & 1.3 & $59 \%$ & $\$ 822$ & $80 \%$ \\
\hline Montana & 1.3 & $69 \%$ & $\$ 908$ & $71 \%$ \\
\hline Nebraska & 1.3 & $82 \%$ & $\$ 887$ & $76 \%$ \\
\hline Nevada & 1.2 & $80 \%$ & $\$ 879$ & $70 \%$ \\
\hline New Hampshire & 1.2 & $85 \%$ & $\$ 930$ & $72 \%$ \\
\hline New Jersey & 1.2 & $79 \%$ & $\$ 901$ & $74 \%$ \\
\hline New Mexico & 1.4 & $77 \%$ & $\$ 776$ & $83 \%$ \\
\hline New York & 1.3 & $80 \%$ & $\$ 988$ & $72 \%$ \\
\hline North Carolina & 1.3 & $70 \%$ & $\$ 924$ & $68 \%$ \\
\hline North Dakota & 1.3 & $86 \%$ & $\$ 881$ & $70 \%$ \\
\hline Ohio & 1.3 & $82 \%$ & $\$ 859$ & $74 \%$ \\
\hline Oklahoma & 1.4 & $56 \%$ & $\$ 774$ & $84 \%$ \\
\hline Oregon & 1.2 & $83 \%$ & $\$ 917$ & $68 \%$ \\
\hline Pennsylvania & 1.3 & $82 \%$ & $\$ 955$ & $70 \%$ \\
\hline Rhode Island & 1.2 & $64 \%$ & $\$ 971$ & $66 \%$ \\
\hline South Carolina & 1.3 & $64 \%$ & $\$ 773$ & $84 \%$ \\
\hline South Dakota & 1.3 & $80 \%$ & $\$ 920$ & $71 \%$ \\
\hline Tennessee & 1.3 & $81 \%$ & $\$ 759$ & $82 \%$ \\
\hline Texas & 1.3 & $81 \%$ & $\$ 668$ & $86 \%$ \\
\hline Utah & 1.3 & $81 \%$ & $\$ 731$ & $82 \%$ \\
\hline Vermont & 1.3 & $60 \%$ & $\$ 1,093$ & $53 \%$ \\
\hline Virginia & 1.3 & $83 \%$ & $\$ 737$ & $85 \%$ \\
\hline Washington & 1.2 & $83 \%$ & $\$ 852$ & $76 \%$ \\
\hline West Virginia & 1.4 & $58 \%$ & $\$ 912$ & $75 \%$ \\
\hline Wisconsin & 1.3 & $64 \%$ & $\$ 1,025$ & $63 \%$ \\
\hline Wyoming & 1.3 & $84 \%$ & $\$ 787$ & $79 \%$ \\
\hline Guam & 2.0 & $50 \%$ & $\$ 871$ & $84 \%$ \\
\hline Virgin Islands & 1.3 & $75 \%$ & $\$ 574$ & $92 \%$ \\
\hline United States & 1.3 & $77 \%$ & $\$ 860$ & $75 \%$ \\
\hline
\end{tabular}

Source: AARP Public Policy Institute analysis of SNAP Quality Control data, 2019 
Benefits among SNAP Households with Adult(s) Ages 50 and Older, 2019

\begin{tabular}{|c|c|c|c|c|c|c|c|c|c|}
\hline \multirow[b]{3}{*}{ State } & \multicolumn{3}{|c|}{ Average SNAP Benefit } & \multicolumn{3}{|c|}{$\begin{array}{l}\text { Receiving Maximum SNAP Benefit } \\
(\%)\end{array}$} & \multicolumn{3}{|c|}{$\begin{array}{l}\text { Receiving Minimum SNAP Benefit } \\
(\%)\end{array}$} \\
\hline & $\begin{array}{l}\text { Households } \\
\text { with Adult(s) }\end{array}$ & $\begin{array}{l}\text { Households } \\
\text { with Adult(s) }\end{array}$ & $\begin{array}{l}\text { Households } \\
\text { with Adult(s) }\end{array}$ & $\begin{array}{c}\text { Households } \\
\text { with Adult(s) }\end{array}$ & $\begin{array}{l}\text { Households } \\
\text { with Adult(s) }\end{array}$ & $\begin{array}{l}\text { Households } \\
\text { with Adult(s) }\end{array}$ & $\begin{array}{l}\text { Households } \\
\text { with Adult(s) }\end{array}$ & $\begin{array}{l}\text { Households } \\
\text { with Adult(s) }\end{array}$ & $\begin{array}{l}\text { Households } \\
\text { ) with Adult(s) }\end{array}$ \\
\hline & $50+$ & 50-59 & $60+$ & $50+$ & 50-59 & $60+$ & $50+$ & $50-59$ & $60+$ \\
\hline Alabama & $\$ 127$ & $\$ 161$ & $\$ 98$ & $19 \%$ & $33 \%$ & $5 \%$ & $17 \%$ & $10 \%$ & $23 \%$ \\
\hline Alaska & $\$ 275$ & $\$ 373$ & $\$ 229$ & $30 \%$ & $41 \%$ & $21 \%$ & $29 \%$ & $15 \%$ & $39 \%$ \\
\hline Arizona & $\$ 140$ & $\$ 190$ & $\$ 103$ & $25 \%$ & $39 \%$ & $13 \%$ & $21 \%$ & $10 \%$ & $29 \%$ \\
\hline Arkansas & $\$ 112$ & $\$ 150$ & $\$ 78$ & $19 \%$ & $31 \%$ & $8 \%$ & $28 \%$ & $15 \%$ & $41 \%$ \\
\hline California & $\$ 172$ & $\$ 216$ & $\$ 140$ & $44 \%$ & $58 \%$ & $34 \%$ & $12 \%$ & $7 \%$ & $16 \%$ \\
\hline Colorado & $\$ 130$ & $\$ 174$ & $\$ 107$ & $24 \%$ & $33 \%$ & $18 \%$ & $23 \%$ & $12 \%$ & $29 \%$ \\
\hline Connecticut & $\$ 152$ & $\$ 172$ & $\$ 143$ & $47 \%$ & $58 \%$ & $41 \%$ & $14 \%$ & $9 \%$ & $17 \%$ \\
\hline Delaware & $\$ 148$ & $\$ 183$ & $\$ 104$ & $27 \%$ & $29 \%$ & $23 \%$ & $22 \%$ & $12 \%$ & $35 \%$ \\
\hline District of Columbia & $\$ 134$ & $\$ 175$ & $\$ 99$ & $35 \%$ & $51 \%$ & $20 \%$ & $19 \%$ & $13 \%$ & $25 \%$ \\
\hline Florida & \$144 & $\$ 194$ & $\$ 122$ & $25 \%$ & $42 \%$ & $17 \%$ & $18 \%$ & $12 \%$ & $22 \%$ \\
\hline Georgia & $\$ 136$ & $\$ 191$ & $\$ 102$ & $25 \%$ & $39 \%$ & $16 \%$ & $20 \%$ & $9 \%$ & $28 \%$ \\
\hline Hawaii & $\$ 306$ & $\$ 389$ & $\$ 270$ & $15 \%$ & $25 \%$ & $10 \%$ & $1 \%$ & $1 \%$ & $1 \%$ \\
\hline Idaho & $\$ 132$ & $\$ 177$ & $\$ 97$ & $16 \%$ & $25 \%$ & $8 \%$ & $17 \%$ & $9 \%$ & $23 \%$ \\
\hline Illinois & $\$ 144$ & $\$ 177$ & $\$ 122$ & $31 \%$ & $42 \%$ & $22 \%$ & $16 \%$ & $8 \%$ & $21 \%$ \\
\hline Indiana & $\$ 126$ & $\$ 156$ & $\$ 102$ & $24 \%$ & $36 \%$ & $14 \%$ & $20 \%$ & $14 \%$ & $25 \%$ \\
\hline lowa & $\$ 121$ & $\$ 158$ & $\$ 85$ & $24 \%$ & $39 \%$ & $7 \%$ & $24 \%$ & $13 \%$ & $36 \%$ \\
\hline Kansas & $\$ 128$ & $\$ 162$ & $\$ 104$ & $20 \%$ & $30 \%$ & $11 \%$ & $18 \%$ & $13 \%$ & $21 \%$ \\
\hline Kentucky & $\$ 129$ & $\$ 178$ & $\$ 83$ & $19 \%$ & $33 \%$ & $5 \%$ & $17 \%$ & $9 \%$ & $25 \%$ \\
\hline Louisiana & $\$ 135$ & $\$ 149$ & $\$ 120$ & $24 \%$ & $35 \%$ & $11 \%$ & $10 \%$ & $9 \%$ & $11 \%$ \\
\hline Maine & $\$ 126$ & $\$ 151$ & $\$ 112$ & $21 \%$ & $31 \%$ & $15 \%$ & $25 \%$ & $20 \%$ & $29 \%$ \\
\hline Maryland & $\$ 120$ & $\$ 163$ & $\$ 96$ & $21 \%$ & $33 \%$ & $14 \%$ & $24 \%$ & $13 \%$ & $30 \%$ \\
\hline Massachusetts & $\$ 152$ & $\$ 178$ & $\$ 142$ & $35 \%$ & $41 \%$ & $32 \%$ & $13 \%$ & $9 \%$ & $14 \%$ \\
\hline Michigan & $\$ 133$ & $\$ 155$ & $\$ 114$ & $28 \%$ & $34 \%$ & $22 \%$ & $18 \%$ & $15 \%$ & $20 \%$ \\
\hline Minnesota & $\$ 109$ & $\$ 131$ & $\$ 91$ & $16 \%$ & $23 \%$ & $10 \%$ & $30 \%$ & $27 \%$ & $33 \%$ \\
\hline Mississippi & $\$ 113$ & $\$ 145$ & $\$ 82$ & $20 \%$ & $31 \%$ & $7 \%$ & $18 \%$ & $10 \%$ & $28 \%$ \\
\hline Missouri & $\$ 127$ & $\$ 157$ & $\$ 106$ & $19 \%$ & $33 \%$ & $7 \%$ & $17 \%$ & $10 \%$ & $24 \%$ \\
\hline Montana & $\$ 132$ & $\$ 165$ & $\$ 112$ & $25 \%$ & $36 \%$ & $17 \%$ & $18 \%$ & $11 \%$ & $23 \%$ \\
\hline Nebraska & $\$ 119$ & $\$ 161$ & $\$ 93$ & $18 \%$ & $29 \%$ & $9 \%$ & $21 \%$ & $10 \%$ & $29 \%$ \\
\hline Nevada & $\$ 114$ & $\$ 157$ & $\$ 87$ & $25 \%$ & $44 \%$ & $13 \%$ & $27 \%$ & $11 \%$ & $37 \%$ \\
\hline New Hampshire & $\$ 129$ & $\$ 146$ & $\$ 115$ & $26 \%$ & $31 \%$ & $21 \%$ & $15 \%$ & $13 \%$ & $16 \%$ \\
\hline New Jersey & $\$ 147$ & $\$ 161$ & $\$ 146$ & $30 \%$ & $36 \%$ & $27 \%$ & $13 \%$ & $20 \%$ & $10 \%$ \\
\hline New Mexico & $\$ 134$ & $\$ 162$ & $\$ 109$ & $25 \%$ & $34 \%$ & $14 \%$ & $20 \%$ & $13 \%$ & $28 \%$ \\
\hline New York & $\$ 178$ & $\$ 204$ & $\$ 166$ & $51 \%$ & $51 \%$ & $50 \%$ & $9 \%$ & $9 \%$ & $10 \%$ \\
\hline North Carolina & $\$ 118$ & $\$ 162$ & $\$ 89$ & $20 \%$ & $32 \%$ & $11 \%$ & $27 \%$ & $18 \%$ & $33 \%$ \\
\hline North Dakota & $\$ 142$ & $\$ 180$ & $\$ 128$ & $27 \%$ & $37 \%$ & $21 \%$ & $19 \%$ & $11 \%$ & $23 \%$ \\
\hline Ohio & $\$ 141$ & $\$ 188$ & $\$ 104$ & $30 \%$ & $44 \%$ & $18 \%$ & $23 \%$ & $12 \%$ & $32 \%$ \\
\hline Oklahoma & $\$ 129$ & $\$ 175$ & $\$ 86$ & $24 \%$ & $35 \%$ & $12 \%$ & $20 \%$ & $11 \%$ & $29 \%$ \\
\hline Oregon & $\$ 131$ & $\$ 173$ & $\$ 106$ & $24 \%$ & $40 \%$ & $14 \%$ & $19 \%$ & $9 \%$ & $26 \%$ \\
\hline Pennsylvania & $\$ 141$ & $\$ 171$ & $\$ 124$ & $29 \%$ & $40 \%$ & $22 \%$ & $15 \%$ & $9 \%$ & $19 \%$ \\
\hline Rhode Island & $\$ 131$ & $\$ 146$ & $\$ 123$ & $27 \%$ & $32 \%$ & $25 \%$ & $21 \%$ & $17 \%$ & $23 \%$ \\
\hline South Carolina & $\$ 121$ & $\$ 157$ & $\$ 91$ & $21 \%$ & $30 \%$ & $13 \%$ & $19 \%$ & $13 \%$ & $24 \%$ \\
\hline South Dakota & $\$ 162$ & $\$ 188$ & $\$ 142$ & $31 \%$ & $38 \%$ & $26 \%$ & $6 \%$ & $6 \%$ & $6 \%$ \\
\hline Tennessee & $\$ 124$ & $\$ 171$ & $\$ 87$ & $24 \%$ & $39 \%$ & $9 \%$ & $27 \%$ & $13 \%$ & $39 \%$ \\
\hline Texas & $\$ 133$ & $\$ 165$ & $\$ 112$ & $17 \%$ & $25 \%$ & $11 \%$ & $11 \%$ & $7 \%$ & $14 \%$ \\
\hline Utah & $\$ 137$ & $\$ 180$ & $\$ 103$ & $28 \%$ & $37 \%$ & $20 \%$ & $21 \%$ & $16 \%$ & $26 \%$ \\
\hline Vermont & $\$ 158$ & $\$ 190$ & $\$ 140$ & $42 \%$ & $49 \%$ & $38 \%$ & $13 \%$ & $11 \%$ & $14 \%$ \\
\hline Virginia & $\$ 130$ & $\$ 180$ & $\$ 87$ & $24 \%$ & $41 \%$ & $8 \%$ & $21 \%$ & $10 \%$ & $29 \%$ \\
\hline Washington & $\$ 135$ & $\$ 158$ & $\$ 124$ & $24 \%$ & $35 \%$ & $17 \%$ & $13 \%$ & $11 \%$ & $13 \%$ \\
\hline West Virginia & $\$ 111$ & $\$ 136$ & $\$ 86$ & $16 \%$ & $24 \%$ & $9 \%$ & $29 \%$ & $20 \%$ & $37 \%$ \\
\hline Wisconsin & $\$ 103$ & $\$ 133$ & $\$ 82$ & $21 \%$ & $32 \%$ & $13 \%$ & $34 \%$ & $24 \%$ & $42 \%$ \\
\hline Wyoming & $\$ 136$ & $\$ 200$ & $\$ 70$ & $18 \%$ & $29 \%$ & $9 \%$ & $25 \%$ & $10 \%$ & $40 \%$ \\
\hline Guam & $\$ 373$ & $\$ 495$ & $\$ 287$ & $39 \%$ & $46 \%$ & $33 \%$ & $1 \%$ & $0 \%$ & $2 \%$ \\
\hline Virgin Islands & $\$ 217$ & $\$ 304$ & $\$ 180$ & $26 \%$ & $51 \%$ & $14 \%$ & $8 \%$ & $4 \%$ & $10 \%$ \\
\hline United States & $\$ 142$ & $\$ 178$ & $\$ 120$ & $29 \%$ & $39 \%$ & $22 \%$ & $17 \%$ & $11 \%$ & $21 \%$ \\
\hline
\end{tabular}

Note: SNAP households with adults 50+, 50-59, and 60+ refer to households with at least one eligible adult 50+, 50-59, and 60+, respectively. Source: AARP Public Policy Institute analysis of SNAP Quality Control data, 2019 
TABLE 4

Share of SNAP Households with Adult(s) Ages 60 and Older Claiming Medical Expense Deduction, 2019

\begin{tabular}{|c|c|c|}
\hline \multirow[b]{2}{*}{ State } & \multicolumn{2}{|c|}{ Households Claiming Deduction } \\
\hline & Number & Percent \\
\hline Alabama & 15,318 & $19 \%$ \\
\hline Alaska & 508 & $6 \%$ \\
\hline Arizona & 8,588 & $9 \%$ \\
\hline Arkansas & 3,263 & $11 \%$ \\
\hline California & 42,115 & $11 \%$ \\
\hline Colorado & 8,496 & $13 \%$ \\
\hline Connecticut & 4,598 & $7 \%$ \\
\hline Delaware & 1,203 & $8 \%$ \\
\hline District of Columbia & 650 & $4 \%$ \\
\hline Florida & 59,191 & $10 \%$ \\
\hline Georgia & 34,913 & $22 \%$ \\
\hline Hawaii & 3,392 & $13 \%$ \\
\hline Idaho & 2,458 & $16 \%$ \\
\hline Illinois & 37,729 & $17 \%$ \\
\hline Indiana & 4,804 & $8 \%$ \\
\hline lowa & 5,065 & $19 \%$ \\
\hline Kansas & 4,587 & $21 \%$ \\
\hline Kentucky & 4,960 & $9 \%$ \\
\hline Louisiana & 6,008 & $8 \%$ \\
\hline Maine & 1,088 & $4 \%$ \\
\hline Maryland & 15,823 & $14 \%$ \\
\hline Massachusetts & 28,803 & $19 \%$ \\
\hline Michigan & 27,742 & $17 \%$ \\
\hline Minnesota & 5,969 & $12 \%$ \\
\hline Mississippi & 5,052 & $11 \%$ \\
\hline Missouri & 18,942 & $25 \%$ \\
\hline Montana & 2,625 & $19 \%$ \\
\hline Nebraska & 3,160 & $19 \%$ \\
\hline Nevada & 6,830 & $12 \%$ \\
\hline New Hampshire & 1,059 & $11 \%$ \\
\hline New Jersey & 20,827 & $14 \%$ \\
\hline New Mexico & 2,052 & $5 \%$ \\
\hline New York & 132,627 & $22 \%$ \\
\hline North Carolina & 28,603 & $18 \%$ \\
\hline North Dakota & 2,492 & $41 \%$ \\
\hline Ohio & 18,488 & $10 \%$ \\
\hline Oklahoma & 4,758 & $9 \%$ \\
\hline Oregon & 19,753 & $18 \%$ \\
\hline Pennsylvania & 46,642 & $16 \%$ \\
\hline Rhode Island & 6,568 & $22 \%$ \\
\hline South Carolina & 10,269 & $16 \%$ \\
\hline South Dakota & 2,444 & $28 \%$ \\
\hline Tennessee & 14,633 & $15 \%$ \\
\hline Texas & 36,206 & $10 \%$ \\
\hline Utah & 879 & $8 \%$ \\
\hline Vermont & 4,664 & $33 \%$ \\
\hline Virginia & 11,211 & $13 \%$ \\
\hline Washington & 13,233 & $10 \%$ \\
\hline West Virginia & 6,250 & $14 \%$ \\
\hline Wisconsin & 23,445 & $29 \%$ \\
\hline Wyoming & 799 & $36 \%$ \\
\hline Guam & 1,013 & $27 \%$ \\
\hline Virgin Islands & 2,394 & $69 \%$ \\
\hline United States & 775,187 & $15 \%$ \\
\hline
\end{tabular}




\section{Appendix B: Maximum SNAP Benefits}

\section{TABLE 1}

Changes in SNAP Maximum Monthly Allotment, 2018-22

\begin{tabular}{|c|c|c|c|c|c|}
\hline \multirow[b]{2}{*}{ Household Size } & \multicolumn{5}{|c|}{ Maximum Monthly SNAP Allotment } \\
\hline & $\begin{array}{l}\text { Oct. 2018- } \\
\text { Sept. } 2019\end{array}$ & $\begin{array}{l}\text { Oct. 2019- } \\
\text { Sept. } 2020\end{array}$ & $\begin{array}{l}\text { Oct. 2020- } \\
\text { Dec. 2020* }\end{array}$ & $\begin{array}{l}\text { Jan. 2021- } \\
\text { Sept. } 2021\end{array}$ & $\begin{array}{l}\text { Oct. 2021- } \\
\text { Sept. 2022** }\end{array}$ \\
\hline 1 & $\$ 192$ & $\$ 194$ & $\$ 204$ & $\$ 234$ & $\$ 250$ \\
\hline 2 & $\$ 353$ & $\$ 355$ & $\$ 374$ & $\$ 430$ & $\$ 459$ \\
\hline 3 & $\$ 505$ & $\$ 509$ & $\$ 535$ & $\$ 616$ & $\$ 658$ \\
\hline 4 & $\$ 642$ & $\$ 646$ & $\$ 680$ & $\$ 782$ & $\$ 835$ \\
\hline 5 & $\$ 762$ & $\$ 768$ & $\$ 807$ & $\$ 929$ & $\$ 992$ \\
\hline 6 & $\$ 914$ & $\$ 921$ & $\$ 969$ & $\$ 1,114$ & $\$ 1,190$ \\
\hline 7 & $\$ 1,011$ & $\$ 1,018$ & $\$ 1,071$ & $\$ 1,232$ & $\$ 1,316$ \\
\hline 8 & $\$ 1,155$ & $\$ 1,164$ & $\$ 1,224$ & $\$ 1,408$ & $\$ 1,504$ \\
\hline Each additional person & $\$ 144$ & $\$ 146$ & $\$ 153$ & $\$ 176$ & $\$ 188$ \\
\hline
\end{tabular}

Note: The allotments described here are for households in the 48 contiguous states and the District of Columbia. The allotments are different in Alaska, Guam, Hawaii, and the Virgin Islands.

1 “Definitions of Food Insecurity," US Department of Agriculture, September 2021, https://www.ers.usda.gov/topics/food-nutritionassistance/food-security-in-the-us/definitions-of-food-security.aspx.

2 Laura J. Samuel et al., "Does the Supplemental Nutrition Assistance Program Affect Hospital Utilization among Older Adults? The Case of Maryland," Population Health Management 21, no. 2 (April 2018): 88-95, https://www.ncbi.nlm.nih.gov/pmc/articles/ PMC5906726/.

3 “SNAP Data Tables," US Department of Agriculture, September 2021, https://www.fns.usda.gov/pd/supplemental-nutritionassistance-program-snap.

4 AARP Public Policy Institute analysis of Current Population Survey Food Security Supplement, 2020.

5 Although the US Department of Agriculture defines elderly as ages 60 and older, this Fact Sheet generally uses the term older households to refer to SNAP households with adults ages 50 and older. Throughout the paper, references to households with older adults mean SNAP households with at least one older adult who is eligible for SNAP.

6 For eligibility purposes, individuals who are ages 60 or older and are unable to purchase food and prepare meals with others in the household because of a permanent disability may be considered a separate SNAP household if the aggregate income of the other individuals they live with is less than 165 percent of the federal poverty level for their household size.

7 While most households with older adults are single-person households, some households have two or more people, raising the average to 1.3 persons per household.

8 Brynne Keith-Jennings and Raheem Chaudhry, "Most Working-Age SNAP Participants Work, but Often in Unstable Jobs," Center on Budget and Policy Priorities, March 15, 2018, https://www.cbpp.org/research/food-assistance/most-working-age-snapparticipants-work-but-often-in-unstable-jobs. 
9 Alisha Coleman-Jensen and Mark Nord, "Disability Is an Important Risk Factor for Food Insecurity," US Department of Agriculture, Economic Research Service, May 6, 2013, https://www.ers.usda.gov/amber-waves/2013/may/disability-is-an-important-risk-factorfor-food-insecurity\#: :text=Not\%20only\%20is\%20food\%20insecurity,than\%20other\%20food\%2Dinsecure\%20households.

10 Disability data are available only for adults under age 60 and likely undercount the true number of individuals with disabilities. Nonelderly individuals are identified as having a disability based on receipt of Supplemental Security Income (SSI) or a combination of hours worked, work registration status, receipt of Social Security, veterans' benefits, or workers' compensation, and/or unit medical expense deduction.

11 Stacy Dean, “Thrifty Food Plan Re-evaluation Puts Nutrition in Reach for SNAP Participants," US Department of Agriculture, August 30, 2021, https://www.usda.gov/media/blog/2021/08/30/thrifty-food-plan-re-evaluation-puts-nutrition-reach-snap-participants.

12 Net income refers to gross income minus allowable deductions. For example, elderly or disabled members may deduct medical expenses that are over $\$ 35$ for the month. More information on deductions is available at "SNAP Eligibility," US Department of Agriculture, October 1, 2021, https://www.fns.usda.gov/snap/recipient/eligibility.

13 There are differences in average monthly allotments by state, impacted largely by household size. In addition, because of the high cost of living in Alaska, Guam, Hawaii, and the Virgin Islands, these states and territories have different income eligibility requirements and benefit maximums.

14 The maximum benefit is higher in Alaska, Guam, Hawaii, and the Virgin Islands due to higher cost of living.

15 “Food Insecurity in the United States," Feeding America, 2021, https://map.feedingamerica.org/.

16 The minimum benefit is higher in Alaska, Guam, Hawaii, and the Virgin Islands due to higher cost of living. See "Cost of Living Adjustment (COLA) Information," US Department of Agriculture, October 1, 2021, https://www.fns.usda.gov/snap/allotment/COLA.

17 The Consolidated Appropriations Act, enacted in December 2020, increased the minimum benefit from January through June 2021. The American Rescue Plan Act, enacted in March 2021, extended this increase through September 2021. The minimum benefit increased to $\$ 20$ in October 2021 as a result of the Thrifty Food Plan reevaluation.

18 Based on the 2019 minimum benefit and average amount spent per meal by people who were food insecure in 2019 (\$3.13).

19 "Maximizing the SNAP Medical Expense Deduction," National Council on Aging, December 2, 2020, https://www.ncoa.org/article/ snap-medical-expense-deduction.

20 Claire Noel-Miller, “Medicare Beneficiaries' Out-of-Pocket Spending for Health Care,” AARP Public Policy Institute, June 11, 2020, https://www.aarp.org/ppi/info-2020/medicare-beneficiaries-out-of-pocket-spending-for-health-care.html.

21 Olivia Dean and Lynda Flowers, “As Pandemic Wears On, Food Challenges Are Increasing for Many Older Americans," AARP Public Policy Institute, July 1, 2020, https://blog.aarp.org/thinking-policy/as-pandemic-wears-on-food-challenges-are-increasing-formany-older-americans.

22 In March 2020, the Families First Coronavirus Response Act allowed USDA to grant state waiver requests for emergency SNAP allotments. All states used these waivers to increase households' SNAP allotments up to the maximum amount allowed for their household size. However, those already receiving the maximum benefit-the lowest-income households-did not receive an increase. USDA took action to remedy this, and beginning April 2021, all SNAP households were eligible to receive emergency allotments. Additionally, the Consolidated Appropriations Act of 2021 increased maximum allotments by 15 percent from the June 2020 value of the TFP for January through June 2021. The American Rescue Plan Act of 2021 further extended this maximum allotment increase through September 2021. While the temporary 15 percent benefit increase ended after September 2021, this loss was offset by a recent reevaluation of the TFP, which resulted in permanent benefit increases for nearly all SNAP participants beginning in October 2021. As a result, SNAP households saw a modest increase in benefits in October: about $\$ 12$ to $\$ 16$ per person per month.

23 “SNAP Data Tables," US Department of Agriculture, September 2021, https://www.fns.usda.gov/pd/supplemental-nutrition-assistance-program-snap.

24 The 2021 data reflect preliminary data from USDA as of October 1, 2021.

Fact Sheet 1275402, December 2021

(c) AARP PUBLIC POLICY INSTITUTE $601 \mathrm{E}$ Street, NW

Washington DC 20049

Follow us on Twitter @AARPpolicy on facebook.com/AARPpolicy www.aarp.org/ppi

For more reports from the Public Policy Institute, visit http://www.aarp.org/ppi/.

https://doi.org/10.26419/ppi.00106.002

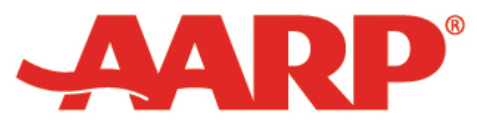

